\title{
Identification and classification of effective factors on realization of entrepreneur government through application of fuzzy TOPSIS
}

\author{
Vahideh Bamshad*
}

Department of Management, Allame Tabatabai University, Tehran, Iran CHRON I C E A B T R A T

Article history:

Received January 20, 2014

Accepted 30 August 2014

Available online

September 102014

Entrepreneur Government

Features of Entrepreneur

Government

Effective Factors on Realization of

Entrepreneur Government

Fuzzy TOPSIS

\begin{abstract}
Entrepreneurship is considered as the key factor of economic growth and development in modern day. Entrepreneurship takes place either individually or organizationally and they normally undertake the risk of entrepreneurship. Entrepreneurs experience the most severe conditions when they deal with a government, which is completely unfamiliar with some rules. If a government supports an entrepreneur one with the required characteristics, then entrepreneurship is facilitated largely for both individuals and organizations. This article provides definitions, features and the effective factors on realization of an entrepreneur government with respect to significance of such a government. To this end, a list of factors that may lead to realization of entrepreneur government was derived from previous studies and then the fuzzy TOPSIS method and collected opinions of experts were applied to facilitate the determination of each of these factors. Finally, a list of influential factors was presented with regards to effective factors on realization of entrepreneur government.
\end{abstract}

\section{Introduction}

After the World War II, the state organization turned into an important means whereby all the governments in the world were looking for economic development in order to achieve particular social objectives (Ahmadpour \& Moghimi, 2001). Therefore, the state organizations play essential role in development process of many countries especially the developing countries. However, many of these organizations have lost their efficiencies in the third world countries in terms of responding to the diverse and increasing needs for citizens (Bygrave \& Hofer, 1991). This is because of budget restrictions, inefficient encouragement and punishment systems, bureaucratic and inflexible structures, lack of motivation among staff and managers as well as ineffective interaction of these organizations with the environment and citizens have led to state organizations encountering serious challenges (Kirzner, 1978). There have been some studies on alterations in government organization and services and they have suggested a new conceptual framework called "entrepreneur government" or "entrepreneurship management in public sector" as the basis of government management, which may help the state organizations in dealing with challenges (Mierlo, 1996). In recent years, the *Corresponding author.

E-mail addresses: Bamshad vahideh@yahoo.com (V.Bamshad) 
entrepreneur in government affairs must be able to change the guideline tendencies in political and administrative environment through preparation of new information regarding political means and objectives such that resources could be altered or allocated through new production techniques and distribution of new products. This article studies the effective factors on realization of entrepreneur government. Hence, the relevant literature would be discussed and then the identified factors would be presented with respect to the research background. Finally, fuzzy TOPSIS method would be used to classify the identified factors.

\section{Literature Review}

\subsection{Entrepreneurship and Entrepreneur}

The term entrepreneurship is widely applied in daily conversation and it is a technical terms used in management and economics. Entrepreneurship is a topic of interest for all communities. Today, organizations require an entrepreneur manager by relying on whose unique characteristics they may overtake other competitors (Lounsbury, 1998). Entrepreneurship includes the process of creating something new while accepting its accompanying risks and benefits. In other words, entrepreneurship is the process of creating something new and valuable accomplished through designation of required time and effort and by taking into account the financial, mental and social risks and the individual and financial satisfaction (Lynch \& Cruise, 2006). Several common aspects are present in all entrepreneurship definitions: risk-taking, creativity, independence and reward. Entrepreneurs are champions of the modern world who are of greater prominence compared with others in the fields of management and economics as they establish new products and benefits in global markets and thus it is virtually impossible to overlook them in spite of the numerous risks they pose (Prokopenko \& Pavlin, 1991). Entrepreneurs do not observe the system the way it is but look at it as it should be. An entrepreneur is a person who organizes various elements and yet designates a separate essence for each of them. Entrepreneurs organize fundamental changes in the world even beyond national boundaries, a person who manipulates production elements and organizes market activities through acquisition and publication of new data. Schumpeter (1934) analyzed the role of entrepreneur in dynamism of market system. In his view, an entrepreneur must be able to identify innovative opportunities, determine the benefits of these opportunities and convince people to invest their resources in the suggested innovation though his/her charisma. An entrepreneur is a person who looks for creative evolution and Schumpeter considered an entrepreneur as a creative destroyer. An entrepreneur is a person who affects the choice of others through acquisition and publication of data on resources and objectives and hence any person may be an entrepreneur. Generally, there are three main economic approaches in entrepreneurship: neoclassic school, Austrian school and Schumpeter school. In neoclassic school, entrepreneur is a lightning calculator, a person who examines and chooses the best option and generative processes in an instance. Austrian school is a more enriched and dynamic concept of entrepreneurship. According to this approach, the entrepreneurs discover the opportunities within an unbalanced market and utilize them until the market reaches timely balance. Schumpeter (1934) considered entrepreneurs as innovative, intrepid and inspirational leaders who may cause economic unbalance through modulation of economic resources into a modern framework.

\subsection{Entrepreneur Government}

According to Drucker (1970), the principles of entrepreneur management in each organization whether private or public are applicable and such management has significantly developed the coordination and cooperation of organizations. Osborne and Gabbler (1992) and Osborne et al. (1998) established identical conditions of entrepreneurship. They believe in a particular model of government called entrepreneur government. Government entrepreneur describes the application of resources through new techniques in order to maximize efficiency and operationalization in public sector organizations. The dry and inflexible bureaucracy in public sector hampers appropriate 
provision of services. The subject of entrepreneurship in public sector was brought up differently from commercial sector since the mid1990s, which led to establishment of a framework for emergence of entrepreneurship. Entrepreneur government is a paradigm against bureaucracy, a government with extraordinarily flexible and compatible organizations (Wickham, 2000). They not only serve the citizens but also empower them. They intensify competition among suppliers and empower citizens through transmission of supervision from bureaucratic organization to the community. Performances are evaluated in an entrepreneur government but their assessment does not include the input but the output. An entrepreneur government is driven by missions and objectives and not by rules and regulations (Say, 1836). They considered customers as their clients, distributed various options to them as well as the chance to choose from among different types of schools, and curricula. An entrepreneur government tries to avoid encumbrance instead of providing required services after occurrence of problems. They exert all their abilities for production and not only for income. An entrepreneur government welcomes distribution power and collaborative management. Market mechanisms are preferred to bureaucratic mechanisms. An entrepreneur government does not seek provision of services but instead all private and public sectors are encouraged to take measures in resolution of social issues (Sadler, 2000). Knowledge of needs of entrepreneurs and the occupations requiring support suggests that the government is in the best position to determine the policy and plans targeted at entrepreneurship. Entrepreneurship is a manageable procedure with key aspects of innovation, risk-taking and effective behavior. Governments may have three responses in the face problems and rapid environmental changes.

First response: extermination of government and total retreat from market and pricing mechanisms. Incapability of government is the proper provision for submission to the market incantation.

Second response: political control over government and bureaucracy where political reformists try to resolve this problem through intensification of external control such as improvement of legislative body (council), better control of ministries by ministers, improvement of financial management and systematic analysis of guidelines.

Third response: an entrepreneur who reforms the public bureaucracy hypotheses. Government entrepreneurship is a solution to break away from market incantation from the one hand bureaucratization from the other hand through injection of competition mechanisms and democratic control of public organizations. Entrepreneurship of government includes essential change on public sector systems and organizations in order to achieve prominent increase in efficiency, consistency and innovative capacity of those systems. This transformation would be achieved through alteration in motivation objective, responsibility, culture and power structure. Entrepreneur government replaces entrepreneur systems with bureaucratic systems, a sort of creating systems and organizations without the need for external pressure.

\subsection{Features of Entrepreneur Government}

These features may be enumerated as follows (Radfar \& Saeidikia, 2010; Radfar et al., 2010):

- Establishing competition between service providing organizations,

- Empowering citizens (instead of serving them) through expulsion of public bureaucracy and its delegation to the community,

- Mission-orientation instead of command-orientation and dominance-orientation,

- Client considered as a customer whose satisfaction must be met,

- Preventing problems instead of providing services after occurrence of problems,

- Focusing on income instead of costs or in other words, concentrating more on opportunities instead of threats,

- Collaborative management spirit in the government, 
- Boosting and supporting private organizations,

- Veneration of and tribute to citizens,

- Minority, lack of concentration and government bureaucracy,

- Interest and enthusiasm to succeed in creative activities and private sector,

- Preventing transmission of costs to other community groups and removing public sector monopoly.

\subsection{Effective Factors on Realization of Entrepreneur Government}

These factors were identified because of study of relevant articles and research and interviews with experts.

1. Economic growth,

2. Coherence of required practical mechanisms,

3. Providing people with the right of choosing from government services,

4. Customer-orientations,

5. Specified role of government,

6. Delegation of public sector companies,

7. Delegation of operative tasks to private and public sectors,

8. Expanding information technology,

9. Establishing competition between service providing companies and public organizations,

10. Clarifying the ultimate objective of government,

11. Establishing planning and policy-making organizations,

12. Assessing the performance of state organizations based on their outcomes,

13. Delegation of authority to economic firms,

14. Shifting budget policies from cost-oriented to performance-oriented,

15. Breaking away from traditional thought of cost-oriented government toward income-oriented government,

16. Modification of infrastructures,

17. Modification of structures and institutionalization,

18. Empowering private sector companies

\section{Research Methodology}

This is an applied research in terms of objective while its methodology is of survey type of field studies. Required data and information were collected from library references, articles and other relevant research. Moreover, interviews and questionnaires were used to examine the opinions of experts from executive bodies and public organizations.

\subsection{Population and Sample}

The statistical population consists of experts from executive bodies and government organizations selected through random method. Comments of 6 experts and veterans were used to perform fuzzy TOPSIS calculations.

\subsection{TOPSIS Method}

According to Jahanshahloo et al. (2006), the fuzzy TOPSIS has the following procedure,

Let $A_{1}, A_{2} \ldots A_{m}$ be $m$ alternatives ranked by $k$ decision makers based on $n$ criteria $\left(C_{1}, C_{2} \ldots C_{n}\right) . X_{i j}$ is the rating score of $A_{i}$ associated with $j^{\text {th }}$ criteria and is defined: 
$\mathrm{x}_{\mathrm{ij}} \in\left[\mathrm{x}_{\mathrm{ij}}^{\mathrm{l}}, \mathrm{x}_{\mathrm{ij}}^{\mathrm{u}}\right]$

Weights of criteria are defined as $w_{1}, w_{2} \ldots w_{n}$ where $w_{j}$ is the weight of $c_{j}$.

We can define a multi attribute decision making (MADM) problem with interval numbers briefly in a decision making matrix like the Table 2 (Tzeng \& Huang, 2011).

Step 1: In TOPSIS method with interval numbers, we have to normalize decision making matrix as we show it below:

$\tilde{a}_{i j}^{l}=\frac{x_{i j}^{l}}{\sqrt{\sum_{j=1}^{m}\left(x_{i j}^{l}\right)^{2}+\left(x_{i j}^{u}\right)^{2}}} \quad i=1,2, \ldots, n, \quad j=1,2, \ldots, m$
$\tilde{a}_{i j}^{u}=\frac{x_{i j}^{u}}{\sqrt{\sum_{j=1}^{m}\left(x_{i j}^{l}\right)^{2}+\left(x_{i j}^{u}\right)^{2}}} \quad i=1,2, \ldots, n, \quad j=1,2, \ldots, m$

Now $\left[x_{i j}^{l}, x_{i j}^{u}\right]$ are normalized and the calculated domain $\left[a_{i j}^{l}, a_{i j}^{u}\right]$ belongs to $[0,1]$. Because of differences in importance of each criterion, in the next step we will calculate weighted normalized decision matrix with interval numbers as follows,

$\bar{v}_{i j}^{l}=w_{j} \tilde{a}_{i j}^{l}, i=1,2, \ldots, n \quad, j=1,2, \ldots, m$

$\bar{v}_{i j}^{u}=w_{j} \tilde{a}_{i j}^{u}, i=1,2, \ldots, n, j=1,2, \ldots, m$

where $w_{i}$ is the weight of $i^{\text {th }}$ criterion and $\sum_{i=1}^{n} w_{i}=1$. Then we describe the ideal positive and negative solutions as follows,

$\bar{A}^{+}=\left\{\bar{v}_{1}^{+}, \ldots, \bar{v}_{n}^{+}\right\}=\left\{\left(\max _{j} \bar{v}_{i j}^{u} \mid i \in I\right),\left(\min _{j} \bar{v}_{i j}^{l} \mid i \in J\right)\right\}$

$\bar{A}^{-}=\left\{\bar{v}_{1}^{-}, \ldots, \bar{v}_{n}^{-}\right\}=\left\{\left(\min _{j} \bar{v}_{i j}^{l} \mid i \in I\right),\left(\max _{j} \bar{v}_{i j}^{u} \mid i \in J\right)\right\}$

where $I$ is the benefit criterion and $J$ is associated with cost. Distances of each alternative from positive and negative solutions have to be calculated by the concepts of $n$ dimensions Euclidean distance method:

$\bar{d}_{j}^{+}=\left\{\sum_{i \in I}\left(\bar{v}_{i j}^{l}-\bar{v}_{i}^{+}\right)^{2}+\sum_{i \in J}\left(v_{i j}^{u}-\bar{v}_{i}^{+}\right)^{2}\right\}^{1 / 2}, j=1,2, \ldots, m$

$\bar{d}_{j}^{-}=\left\{\sum_{i \in I}\left(\bar{v}_{i j}^{u}-\bar{v}_{i}^{-}\right)^{2}+\sum_{i \in J}\left(v_{i j}^{l}-\bar{v}_{i}^{-}\right)^{2}\right\}^{1 / 2}, j=1,2, \ldots, m$

For determining rank of each alternative we calculate the closeness coefficient as follows:

$\overline{c l}=\frac{\bar{d}_{j}^{-}}{\bar{d}_{j}^{-}+\bar{d}_{j}^{+}} \quad, j=1,2, \ldots, m$

Based on "closeness coefficient", we can rank alternatives and select the best one.

\section{Research findings}

\subsection{Sieving identified factors}

Having identified the numerous factors that affect the realization of entrepreneur government, this list was submitted to experts and they were asked to score each factor from 1 to 5 . Thus, only those factors with average scores higher than the mean (2.5) are entered the final model. 
Table 1

List of effective factors on realization of entrepreneur government

\begin{tabular}{lll}
\hline Row & effective factors & Average Score \\
\hline 1 & Economic growth & 1.8 \\
2 & Coherence of required practical mechanisms & 2.3 \\
3 & Providing people with the right of choosing from government services & 4.3 \\
4 & Customer-orientations & Merge with No. 3 \\
5 & Specified role of government & 4.4 \\
6 & Delegation of public sector companies & Merge with No. 5 \\
7 & Delegation of operative tasks to private and public sectors & 2.1 \\
8 & Expanding information technology & 3.6 \\
9 & Establishing competition between service providing companies and public organizations & 4.1 \\
10 & Clarifying the ultimate objective of government & 4 \\
11 & Establishing planning and policy-making organizations & 3.5 \\
12 & Assessing the performance of state organizations based on their outcomes & Merge with No. 5 \\
13 & Delegation of authority to economic firms & 4.6 \\
14 & Shifting budget policies from cost-oriented to performance-oriented & Merge with No. 13 \\
15 & Breaking away from traditional thought of cost-oriented government toward income-oriented government & 2.4 \\
16 & Modification of infrastructures & Merge with No. 15 \\
17 & Modification of structures and institutionalization & Merge with No. 5 \\
18 & Empowering private sector companies & Merge with No. 9 \\
\hline
\end{tabular}

Table 2 demonstrates the list of final factors entered into fuzzy TOPSIS model.

\section{Table 2}

Final list of effective factors on realization of entrepreneur government

\begin{tabular}{|c|c|c|}
\hline Row & Final effective factors & Symbol \\
\hline 1 & Providing people with the right of choosing from government services & $\mathrm{EF}_{1}$ \\
\hline 2 & Delegation of public sector companies & $\mathrm{EF}_{2}$ \\
\hline 3 & Establishing competition between service providing companies and public organizations & $\mathrm{EF}_{3}$ \\
\hline 4 & Clarifying the ultimate objective of government & $\mathrm{EF}_{4}$ \\
\hline 5 & Establishing planning and policy-making organizations & $\mathrm{EF}_{5}$ \\
\hline 6 & Assessing the performance of state organizations based on their outcomes & $\mathrm{EF}_{6}$ \\
\hline 7 & Shifting budget policies from cost-oriented to performance-oriented & $\mathrm{EF}_{7}$ \\
\hline
\end{tabular}

\subsection{Identification of Criteria}

Some of the criteria were identified and exploited in order to determine the desirability of each of the identified effective factors. These criteria approved by experts are: feasibility, facility, time, cost, consistency with overall policies of system and risk.

\subsection{Experts' judgment matrix}

After identification of effective factors and criteria, the experts' judgments were asked through questionnaires. A verbal range used to glean experts' opinion. Then answers were changed from verbal range to fuzzy numbers by Chou and Chang method (Chou \& Chang, 2008):

Table 3

Verbal range to fuzzy numbers

\begin{tabular}{ccccccc}
\hline VG & G & MG & F & MP & P & VP \\
\hline$(9,10,10)$ & $(7,9,10)$ & $(5,7,9)$ & $(3,5,7)$ & $(1,3,5)$ & $(0,1,3)$ & $(0,0,1)$ \\
\hline
\end{tabular}

Based on Table 3, the initial matrix is calculated and the results are given in Table 4.

\subsection{Solving Model}

Results are gained from solving the model based on TOPSIS model steps. 
Table 4

Initial Matrix of experts' judgment

\begin{tabular}{|c|c|c|c|c|c|c|c|c|c|c|c|c|c|c|c|c|c|c|c|}
\hline & & \multicolumn{3}{|c|}{$\mathrm{Ex}_{1}$} & \multicolumn{3}{|c|}{$\mathrm{Ex}_{2}$} & \multicolumn{3}{|c|}{$\mathrm{Ex}_{3}$} & \multicolumn{3}{|c|}{$\mathrm{Ex}_{4}$} & \multicolumn{3}{|c|}{$\mathrm{Ex}_{5}$} & \multicolumn{3}{|c|}{$\mathrm{Ex}_{6}$} \\
\hline \multirow{7}{*}{$\mathrm{C}_{1}$} & $\mathrm{EF}_{1}$ & 0 & 1 & 2 & 4 & 5 & 6 & 1 & 2 & 3 & 0 & 1 & 2 & 4 & 5 & 6 & 0 & 1 & 2 \\
\hline & $\mathrm{EF}_{2}$ & 0 & 1 & 2 & 1 & 2 & 3 & 1 & 2 & 3 & 0 & 1 & 2 & 1 & 2 & 3 & 0 & 1 & 2 \\
\hline & $\mathrm{EF}_{3}$ & 2 & 3 & 4 & 4 & 5 & 6 & 2 & 3 & 4 & 5 & 6 & 7 & 4 & 5 & 6 & 5 & 6 & 7 \\
\hline & $\mathrm{EF}_{4}$ & 7 & 8 & 9 & 7 & 8 & 9 & 7 & 8 & 9 & 5 & 6 & 7 & 7 & 8 & 9 & 5 & 6 & 7 \\
\hline & $\mathrm{EF}_{5}$ & 4 & 5 & 6 & 4 & 5 & 6 & 2 & 3 & 4 & 1 & 2 & 3 & 4 & 5 & 6 & 1 & 2 & 3 \\
\hline & $\mathrm{EF}_{6}$ & 5 & 6 & 7 & 4 & 5 & 6 & 4 & 5 & 6 & 5 & 6 & 7 & 4 & 5 & 6 & 5 & 6 & 7 \\
\hline & $\mathrm{EF}_{7}$ & 2 & 3 & 4 & 5 & 6 & 7 & 5 & 6 & 7 & 4 & 5 & 6 & 5 & 6 & 7 & 4 & 5 & 6 \\
\hline \multirow{7}{*}{$\mathrm{C}_{2}$} & $\mathrm{EF}_{1}$ & 1 & 2 & 3 & 1 & 2 & 3 & 2 & 3 & 4 & 2 & 3 & 4 & 2 & 3 & 4 & 1 & 2 & 3 \\
\hline & $\mathrm{EF}_{2}$ & 0 & 1 & 2 & 1 & 2 & 3 & 1 & 2 & 3 & 0 & 1 & 2 & 1 & 2 & 3 & 0 & 1 & 2 \\
\hline & $\mathrm{EF}_{3}$ & 2 & 3 & 4 & 4 & 5 & 6 & 4 & 5 & 6 & 1 & 2 & 3 & 4 & 5 & 6 & 2 & 3 & 4 \\
\hline & $\mathrm{EF}_{4}$ & 4 & 5 & 6 & 4 & 5 & 6 & 5 & 6 & 7 & 5 & 6 & 7 & 5 & 6 & 7 & 4 & 5 & 6 \\
\hline & $\mathrm{EF}_{5}$ & 4 & 5 & 6 & 2 & 3 & 4 & 2 & 3 & 4 & 4 & 5 & 6 & 2 & 3 & 4 & 4 & 5 & 6 \\
\hline & $\mathrm{EF}_{6}$ & 7 & 8 & 9 & 5 & 6 & 7 & 5 & 6 & 7 & 5 & 6 & 7 & 5 & 6 & 7 & 7 & 8 & 9 \\
\hline & $\mathrm{EF}_{7}$ & 4 & 5 & 6 & 4 & 5 & 6 & 4 & 5 & 6 & 4 & 5 & 6 & 4 & 5 & 6 & 4 & 5 & 6 \\
\hline \multirow{7}{*}{$\mathrm{C}_{3}$} & $\mathrm{EF}_{1}$ & 1 & 2 & 3 & 1 & 2 & 3 & 2 & 3 & 4 & 1 & 2 & 3 & 1 & 2 & 3 & 1 & 2 & 3 \\
\hline & $\mathrm{EF}_{2}$ & 1 & 2 & 3 & 1 & 2 & 3 & 0 & 1 & 2 & 1 & 2 & 3 & 1 & 2 & 3 & 1 & 2 & 3 \\
\hline & $\mathrm{EF}_{3}$ & 4 & 5 & 6 & 4 & 5 & 6 & 2 & 3 & 4 & 4 & 5 & 6 & 4 & 5 & 6 & 4 & 5 & 6 \\
\hline & $\mathrm{EF}_{4}$ & 5 & 6 & 7 & 7 & 8 & 9 & 5 & 6 & 7 & 5 & 6 & 7 & 7 & 8 & 9 & 5 & 6 & 7 \\
\hline & $\mathrm{EF}_{5}$ & 2 & 3 & 4 & 2 & 3 & 4 & 1 & 2 & 3 & 2 & 3 & 4 & 2 & 3 & 4 & 2 & 3 & 4 \\
\hline & $\mathrm{EF}_{6}$ & 7 & 8 & 9 & 7 & 8 & 9 & 7 & 8 & 9 & 7 & 8 & 9 & 7 & 8 & 9 & 7 & 8 & 9 \\
\hline & $\mathrm{EF}_{7}$ & 5 & 6 & 7 & 5 & 6 & 7 & 4 & 5 & 6 & 4 & 5 & 6 & 5 & 6 & 7 & 5 & 6 & 7 \\
\hline \multirow{7}{*}{$\mathrm{C}_{4}$} & $\mathrm{EF}_{1}$ & 4 & 5 & 6 & 1 & 2 & 3 & 4 & 5 & 6 & 5 & 6 & 7 & 1 & 2 & 3 & 5 & 6 & 7 \\
\hline & $\mathrm{EF}_{2}$ & 4 & 5 & 6 & 7 & 8 & 9 & 4 & 5 & 6 & 5 & 6 & 7 & 7 & 8 & 9 & 5 & 6 & 7 \\
\hline & $\mathrm{EF}_{3}$ & 4 & 5 & 6 & 4 & 5 & 6 & 5 & 6 & 7 & 5 & 6 & 7 & 4 & 5 & 6 & 5 & 6 & 7 \\
\hline & $\mathrm{EF}_{4}$ & 8 & 9 & 10 & 8 & 9 & 10 & 7 & 8 & 9 & 7 & 8 & 9 & 8 & 9 & 10 & 7 & 8 & 9 \\
\hline & $\mathrm{EF}_{5}$ & 8 & 9 & 10 & 8 & 9 & 10 & 5 & 6 & 7 & 7 & 8 & 9 & 8 & 9 & 10 & 7 & 8 & 9 \\
\hline & $\mathrm{EF}_{6}$ & 8 & 9 & 10 & 7 & 8 & 9 & 7 & 8 & 9 & 5 & 6 & 7 & 7 & 8 & 9 & 5 & 6 & 7 \\
\hline & $\mathrm{EF}_{7}$ & 4 & 5 & 6 & 4 & 5 & 6 & 5 & 6 & 7 & 5 & 6 & 7 & 4 & 5 & 6 & 5 & 6 & 7 \\
\hline \multirow{7}{*}{$\mathrm{C}_{5}$} & $\mathrm{EF}_{1}$ & 1 & 2 & 3 & 1 & 2 & 3 & 1 & 2 & 3 & 5 & 6 & 7 & 1 & 2 & 3 & 1 & 2 & 3 \\
\hline & $\mathrm{EF}_{2}$ & 1 & 2 & 3 & 1 & 2 & 3 & 2 & 3 & 4 & 1 & 2 & 3 & 1 & 2 & 3 & 2 & 3 & 4 \\
\hline & $\mathrm{EF}_{3}$ & 7 & 8 & 9 & 4 & 5 & 6 & 7 & 8 & 9 & 5 & 6 & 7 & 4 & 5 & 6 & 7 & 8 & 9 \\
\hline & $\mathrm{EF}_{4}$ & 7 & 8 & 9 & 7 & 8 & 9 & 5 & 6 & 7 & 7 & 8 & 9 & 7 & 8 & 9 & 5 & 6 & 7 \\
\hline & $\mathrm{EF}_{5}$ & 4 & 5 & 6 & 7 & 8 & 9 & 4 & 5 & 6 & 7 & 8 & 9 & 7 & 8 & 9 & 4 & 5 & 6 \\
\hline & $\mathrm{EF}_{6}$ & 7 & 8 & 9 & 8 & 9 & 10 & 8 & 9 & 10 & 8 & 9 & 10 & 8 & 9 & 10 & 8 & 9 & 10 \\
\hline & $\mathrm{EF}_{7}$ & 5 & 6 & 7 & 5 & 6 & 7 & 5 & 6 & 7 & 5 & 6 & 7 & 5 & 6 & 7 & 5 & 6 & 7 \\
\hline \multirow{7}{*}{$\mathrm{C}_{6}$} & $\mathrm{EF}_{1}$ & 2 & 3 & 4 & 4 & 5 & 6 & 2 & 3 & 4 & 1 & 2 & 3 & 1 & 2 & 3 & 4 & 5 & 6 \\
\hline & $\mathrm{EF}_{2}$ & 4 & 5 & 6 & 4 & 5 & 6 & 4 & 5 & 6 & 0 & 1 & 2 & 0 & 1 & 2 & 4 & 5 & 6 \\
\hline & $\mathrm{EF}_{3}$ & 1 & 2 & 3 & 1 & 2 & 3 & 2 & 3 & 4 & 2 & 3 & 4 & 2 & 3 & 4 & 1 & 2 & 3 \\
\hline & $\mathrm{EF}_{4}$ & 2 & 3 & 4 & 2 & 3 & 4 & 4 & 5 & 6 & 2 & 3 & 4 & 2 & 3 & 4 & 2 & 3 & 4 \\
\hline & $\mathrm{EF}_{5}$ & 4 & 5 & 6 & 4 & 5 & 6 & 2 & 3 & 4 & 4 & 5 & 6 & 4 & 5 & 6 & 4 & 5 & 6 \\
\hline & $\mathrm{EF}_{6}$ & 5 & 6 & 7 & 4 & 5 & 6 & 4 & 5 & 6 & 4 & 5 & 6 & 4 & 5 & 6 & 4 & 5 & 6 \\
\hline & $\mathrm{EF}_{7}$ & 4 & 5 & 6 & 5 & 6 & 7 & 2 & 3 & 4 & 5 & 6 & 7 & 5 & 6 & 7 & 5 & 6 & 7 \\
\hline
\end{tabular}

Table 5

Steps for solving the model

\begin{tabular}{|c|c|c|c|c|c|c|c|c|c|c|c|c|c|}
\hline & & \multicolumn{3}{|c|}{ Final Result } & \multicolumn{3}{|c|}{ Normalized } & \multicolumn{3}{|c|}{ Final Weights } & \multicolumn{3}{|c|}{ Weights*Normalized } \\
\hline \multirow{7}{*}{$\mathrm{C}_{1}$} & $\mathrm{EF}_{1}$ & 0.000 & 2.500 & 6.000 & 0.000 & 0.250 & 0.600 & \multirow{7}{*}{0.760} & \multirow{7}{*}{0.810} & \multirow{7}{*}{0.860} & 0.000 & 0.203 & 0.516 \\
\hline & $\mathrm{EF}_{2}$ & 10.000 & 1.500 & 3.000 & 1.000 & 0.150 & 0.300 & & & & 0.760 & 0.122 & 0.258 \\
\hline & $\mathrm{EF}_{3}$ & 7.000 & 4.667 & 7.000 & 0.700 & 0.467 & 0.700 & & & & 0.532 & 0.378 & 0.602 \\
\hline & $\mathrm{EF}_{4}$ & 2.000 & 7.333 & 9.000 & 0.200 & 0.733 & 0.900 & & & & 0.152 & 0.594 & 0.774 \\
\hline & $\mathrm{EF}_{5}$ & 2.000 & 3.667 & 6.000 & 0.200 & 0.367 & 0.600 & & & & 0.152 & 0.297 & 0.516 \\
\hline & $\mathrm{EF}_{6}$ & 3.000 & 5.500 & 7.000 & 0.300 & 0.550 & 0.700 & & & & 0.228 & 0.446 & 0.602 \\
\hline & $\mathrm{EF}_{7}$ & 3.000 & 5.167 & 7.000 & 0.300 & 0.517 & 0.700 & & & & 0.228 & 0.419 & 0.602 \\
\hline \multirow{7}{*}{$\mathrm{C}_{2}$} & $\mathrm{EF}_{1}$ & 1.000 & 2.500 & 4.000 & 0.111 & 0.278 & 0.444 & \multirow{7}{*}{0.760} & \multirow{7}{*}{0.810} & \multirow{7}{*}{0.860} & 0.084 & 0.225 & 0.382 \\
\hline & $\mathrm{EF}_{2}$ & 0.000 & 1.500 & 3.000 & 0.000 & 0.167 & 0.333 & & & & 0.000 & 0.135 & 0.287 \\
\hline & $\mathrm{EF}_{3}$ & 1.000 & 3.833 & 6.000 & 0.111 & 0.426 & 0.667 & & & & 0.084 & 0.345 & 0.573 \\
\hline & $\mathrm{EF}_{4}$ & 4.000 & 5.500 & 7.000 & 0.444 & 0.611 & 0.778 & & & & 0.338 & 0.495 & 0.669 \\
\hline & $\mathrm{EF}_{5}$ & 2.000 & 4.000 & 6.000 & 0.222 & 0.444 & 0.667 & & & & 0.169 & 0.360 & 0.573 \\
\hline & $\mathrm{EF}_{6}$ & 5.000 & 6.667 & 9.000 & 0.556 & 0.741 & 1.000 & & & & 0.422 & 0.600 & 0.860 \\
\hline & $\mathrm{EF}_{7}$ & 4.000 & 5.000 & 6.000 & 0.444 & 0.556 & 0.667 & & & & 0.338 & 0.450 & 0.573 \\
\hline \multirow{7}{*}{$\mathrm{C}_{3}$} & $\mathrm{EF}_{1}$ & 1.000 & 2.167 & 4.000 & 0.111 & 0.241 & 0.444 & \multirow{7}{*}{0.650} & \multirow{7}{*}{0.700} & \multirow{7}{*}{0.750} & 0.072 & 0.169 & 0.333 \\
\hline & $\mathrm{EF}_{2}$ & 0.000 & 1.833 & 3.000 & 0.000 & 0.204 & 0.333 & & & & 0.000 & 0.143 & 0.250 \\
\hline & $\mathrm{EF}_{3}$ & 2.000 & 4.667 & 6.000 & 0.222 & 0.519 & 0.667 & & & & 0.144 & 0.363 & 0.500 \\
\hline & $\mathrm{EF}_{4}$ & 5.000 & 6.667 & 9.000 & 0.556 & 0.741 & 1.000 & & & & 0.361 & 0.519 & 0.750 \\
\hline & $\mathrm{EF}_{5}^{4}$ & 1.000 & 2.833 & 6.000 & 0.111 & 0.315 & 0.667 & & & & 0.072 & 0.220 & 0.500 \\
\hline & $\mathrm{EF}_{6}$ & 7.000 & 8.000 & 9.000 & 0.778 & 0.889 & 1.000 & & & & 0.506 & 0.622 & 0.750 \\
\hline & $\mathrm{EF}_{7}$ & 4.000 & 5.667 & 7.000 & 0.444 & 0.630 & 0.778 & & & & 0.289 & 0.441 & 0.583 \\
\hline \multirow{7}{*}{$\mathrm{C}_{4}$} & $\mathrm{EF}_{1}$ & 1.000 & 4.333 & 7.000 & 0.100 & 0.433 & 0.700 & \multirow{7}{*}{0.750} & \multirow{7}{*}{0.800} & \multirow{7}{*}{0.850} & 0.075 & 0.347 & 0.595 \\
\hline & $\mathrm{EF}_{2}$ & 4.000 & 6.333 & 9.000 & 0.400 & 0.633 & 0.900 & & & & 0.300 & 0.507 & 0.765 \\
\hline & $\mathrm{EF}_{3}$ & 4.000 & 5.500 & 7.000 & 0.400 & 0.550 & 0.700 & & & & 0.300 & 0.440 & 0.595 \\
\hline & $\mathrm{EF}_{4}$ & 7.000 & 8.500 & 10.000 & 0.700 & 0.850 & 1.000 & & & & 0.525 & 0.680 & 0.850 \\
\hline & $\mathrm{EF}_{5}$ & 5.000 & 8.167 & 10.000 & 0.500 & 0.817 & 1.000 & & & & 0.375 & 0.653 & 0.850 \\
\hline & $\mathrm{EF}_{6}$ & 5.000 & 7.500 & 10.000 & 0.500 & 0.750 & 1.000 & & & & 0.375 & 0.600 & 0.850 \\
\hline & $\mathrm{EF}_{7}$ & 4.000 & 5.500 & 7.000 & 0.400 & 0.550 & 0.700 & & & & 0.300 & 0.440 & 0.595 \\
\hline \multirow{7}{*}{$\mathrm{C}_{5}$} & $\mathrm{EF}_{1}$ & 1.000 & 2.667 & 7.000 & 0.100 & 0.267 & 0.700 & & & & 0.066 & 0.189 & 0.532 \\
\hline & $\mathrm{EF}_{2}$ & 1.000 & 2.333 & 4.000 & 0.100 & 0.233 & 0.400 & & & & 0.066 & 0.166 & 0.304 \\
\hline & $\mathrm{EF}_{3}$ & 4.000 & 6.667 & 9.000 & 0.400 & 0.667 & 0.900 & & & & 0.264 & 0.473 & 0.684 \\
\hline & $\mathrm{EF}_{4}$ & 5.000 & 7.333 & 9.000 & 0.500 & 0.733 & 0.900 & 0.660 & 0.710 & 0.760 & 0.330 & 0.521 & 0.684 \\
\hline & $\mathrm{EF}_{5}$ & 4.000 & 6.500 & 9.000 & 0.400 & 0.650 & 0.900 & & & & 0.264 & 0.462 & 0.684 \\
\hline & $\mathrm{EF}_{6}$ & 7.000 & 8.833 & 10.000 & 0.700 & 0.883 & 1.000 & & & & 0.462 & 0.627 & 0.760 \\
\hline & $\mathrm{EF}_{7}$ & 5.000 & 6.000 & 7.000 & 0.500 & 0.600 & 0.700 & & & & 0.330 & 0.426 & 0.532 \\
\hline & $\mathrm{EF}_{1}$ & 1.000 & 3.500 & 6.000 & 0.143 & 0.500 & 0.857 & & & & 0.104 & 0.390 & 0.711 \\
\hline & $\mathrm{EF}_{2}$ & 0.000 & 4.000 & 6.000 & 0.000 & 0.571 & 0.857 & & & & 0.000 & 0.446 & 0.711 \\
\hline & $\mathrm{EF}_{3}$ & 1.000 & 2.500 & 4.000 & 0.143 & 0.357 & 0.571 & & & & 0.104 & 0.279 & 0.474 \\
\hline $\mathrm{C}_{6}$ & $\mathrm{EF}_{4}$ & 2.000 & 3.500 & 6.000 & 0.286 & 0.500 & 0.857 & 0.730 & 0.780 & 0.830 & 0.209 & 0.390 & 0.711 \\
\hline & $\mathrm{EF}_{5}$ & 2.000 & 4.500 & 6.000 & 0.286 & 0.643 & 0.857 & & & & 0.209 & 0.501 & 0.711 \\
\hline & $\mathrm{EF}_{6}$ & 4.000 & 5.125 & 7.000 & 0.571 & 0.732 & 1.000 & & & & 0.417 & 0.571 & 0.830 \\
\hline & $\mathrm{EF}_{7}$ & 2.000 & 5.125 & 7.000 & 0.286 & 0.732 & 1.000 & & & & 0.209 & 0.571 & 0.830 \\
\hline
\end{tabular}


Positive and negative ideal solutions and alternative distances from each one:

Table 6

Positive and negative ideal solutions

\begin{tabular}{|c|c|c|c|c|c|c|c|c|c|c|c|}
\hline & & $\mathrm{d}+$ & $\mathrm{d}(\mathrm{a}, \mathrm{a}+)$ & d- & $d(a, a-)$ & & & $\mathrm{d}+$ & $\mathrm{d}(\mathrm{a}, \mathrm{a}+)$ & d- & $d(a, a-)$ \\
\hline \multirow{7}{*}{$\mathrm{C}_{1}$} & $\mathrm{EF}_{1}$ & \multirow{7}{*}{0.774} & 0.165 & \multirow{7}{*}{0.000} & 0.051 & \multirow{7}{*}{$\mathrm{C}_{4}$} & $\mathrm{EF}_{1}$ & 0.850 & 0.153 & \multirow{7}{*}{0.075} & 0.057 \\
\hline & $\mathrm{EF}_{2}$ & & 0.115 & & 0.110 & & $\mathrm{EF}_{2}$ & & 0.071 & & 0.119 \\
\hline & $\mathrm{EF}_{3}$ & & 0.041 & & 0.131 & & $\mathrm{EF}_{3}$ & & 0.089 & & 0.076 \\
\hline & $\mathrm{EF}_{4}$ & & 0.070 & & 0.163 & & $\mathrm{EF}_{4}$ & & 0.022 & & 0.195 \\
\hline & $\mathrm{EF}_{5}$ & & 0.113 & & 0.063 & & $\mathrm{EF}_{5}$ & & 0.044 & & 0.171 \\
\hline & $\mathrm{EF}_{6}$ & & 0.073 & & 0.102 & & $\mathrm{EF}_{6}$ & & 0.048 & & 0.161 \\
\hline & $\mathrm{EF}_{7}$ & & 0.076 & & 0.098 & & $\mathrm{EF}_{7}$ & & 0.089 & & 0.076 \\
\hline \multirow{7}{*}{$\mathrm{C}_{2}$} & $\mathrm{EF}_{1}$ & \multirow{7}{*}{0.860} & 0.205 & \multirow{7}{*}{0.000} & 0.034 & \multirow{7}{*}{$\mathrm{C}_{5}$} & $\mathrm{EF}_{1}$ & 0.760 & 0.143 & \multirow{7}{*}{0.066} & 0.039 \\
\hline & $\mathrm{EF}_{2}$ & & 0.266 & & 0.017 & & $\mathrm{EF}_{2}$ & & 0.174 & & 0.011 \\
\hline & $\mathrm{EF}_{3}$ & & 0.158 & & 0.076 & & $\mathrm{EF}_{3}$ & & 0.056 & & 0.098 \\
\hline & $\mathrm{EF}_{4}$ & & 0.074 & & 0.134 & & $\mathrm{EF}_{4}$ & & 0.041 & & 0.110 \\
\hline & $\mathrm{EF}_{5}$ & & 0.135 & & 0.081 & & $\mathrm{EF}_{5}$ & & 0.057 & & 0.096 \\
\hline & $\mathrm{EF}_{6}$ & & 0.043 & & 0.213 & & $\mathrm{EF}_{6}$ & & 0.018 & & 0.159 \\
\hline & $\mathrm{EF}_{7}$ & & 0.087 & & 0.108 & & $\mathrm{EF}_{7}$ & & 0.058 & & 0.069 \\
\hline \multirow{7}{*}{$\mathrm{C}_{3}$} & $\mathrm{EF}_{1}$ & \multirow{7}{*}{0.750} & 0.162 & \multirow{7}{*}{0.000} & 0.024 & \multirow{7}{*}{$\mathrm{C}_{6}$} & $\mathrm{EF}_{1}$ & 0.830 & 0.122 & \multirow{7}{*}{0.000} & 0.112 \\
\hline & $\mathrm{EF}_{2}$ & & 0.197 & & 0.014 & & $\mathrm{EF}_{2}$ & & 0.142 & & 0.117 \\
\hline & $\mathrm{EF}_{3}$ & & 0.096 & & 0.067 & & $\mathrm{EF}_{3}$ & & 0.160 & & 0.052 \\
\hline & $\mathrm{EF}_{4}$ & & 0.034 & & 0.160 & & $\mathrm{EF}_{4}$ & & 0.099 & & 0.117 \\
\hline & $\mathrm{EF}_{5}$ & & 0.134 & & 0.051 & & $\mathrm{EF}_{5}$ & & 0.085 & & 0.134 \\
\hline & $\mathrm{EF}_{6}$ & & 0.013 & & 0.201 & & $\mathrm{EF}_{6}$ & & 0.040 & & 0.198 \\
\hline & $\mathrm{EF}_{7}$ & & 0.056 & & 0.103 & & $\mathrm{EF}_{7}$ & & 0.076 & & 0.176 \\
\hline
\end{tabular}

\subsection{Final Ranking}

Final Rank of effective factors are as below:

Table 7

Final ranks

\begin{tabular}{|c|c|c|c|c|c|}
\hline & $\mathrm{d}+$ & d- & $(d+)+(d-)$ & $(\mathrm{d}-) /(\mathrm{d}+)+(\mathrm{d}-)$ & Ranks \\
\hline $\mathrm{EF}_{1}$ & 1.219 & 1.408 & 2.627 & 0.536 & 3 \\
\hline $\mathrm{EF}_{2}$ & 1.310 & 1.213 & 2.523 & 0.481 & 4 \\
\hline $\mathrm{EF}_{3}$ & 1.415 & 0.948 & 2.363 & 0.401 & 5 \\
\hline $\mathrm{EF}_{4}$ & 0.893 & 1.885 & 2.778 & 0.679 & 1 \\
\hline $\mathrm{EF}_{5}$ & 1.156 & 1.512 & 2.668 & 0.567 & 2 \\
\hline $\mathrm{EF}_{6}$ & 1.975 & 0.862 & 2.837 & 0.304 & 7 \\
\hline $\mathrm{EF}_{7}$ & 1.801 & 0.879 & 2.680 & 0.328 & 6 \\
\hline
\end{tabular}

\section{Conclusion and Discussion}

Government plays the role of leader in the process of development and growth of a society as it directs the development process and interferes whenever deemed necessary. The rethinking in the overall framework of society through a modern perspective is not only a restructuring of objective but also the innovation of new structures. To this end, independent performance of these organizations must become institutionalized and this would not be possible except for the government to alter its form and essence, becoming an entrepreneur government. The final ranking of effective factors is as follows:

Table 8

Results from Fuzzy TOPSIS

\begin{tabular}{|c|c|c|c|}
\hline Row & Final effective factors & Symbol & Final Rank \\
\hline 1 & Providing people with the right of choosing from government services & $\mathrm{EF}_{1}$ & 3 \\
\hline 2 & Delegation of public sector companies & $\mathrm{EF}_{2}$ & 4 \\
\hline 3 & Establishing competition between service providing companies and public organizations & $\mathrm{EF}_{3}$ & 5 \\
\hline 4 & Clarifying the ultimate objective of government & $\mathrm{EF}_{4}$ & 1 \\
\hline 5 & Establishing planning and policy-making organizations & $\mathrm{EF}_{5}$ & 2 \\
\hline 6 & Assessing the performance of state organizations based on their outcomes & $\mathrm{EF}_{6}$ & 7 \\
\hline 7 & Shifting budget policies from cost-oriented to performance-oriented & $\mathrm{EF}_{7}$ & 6 \\
\hline
\end{tabular}


The results suggest that most important factors according to experts are the ultimate objective of government and specified role government for administration, staff and people. In case the ultimate objectives are specified and the role of government is clarified, then reform and movement toward the objective may be expected. The second factor according to experts is to establish planning and policy-making institutions. These institutions study and develop operational plans required for transformation government into an entrepreneur one in form of specialized taskforces. Top entrepreneurs and experts may be used in meetings. Policy-making institutions play an important role in development of operational plans from the follow-up on bill to their ratifications. Edition of strategic plans is one of the activities of such institutions. Integrated entrepreneurship strategy and coherence of executive mechanisms are required to drive the required targets of government. Government must operationalize its targets through coherent and revised plans. This program must include all the subcategories of administration. A strategic plan contributes to continuous control by government. Hence, the government may control the progress of its subcategories within provincial governments and miniseries based on predetermined objectives and take immediate measures in case of any deviation from the set targets. The third factor is the right of choice among public organizations and people. When people are able to choose their desirable option from the public organizations, then the competition among state organizations would also be increased. Hence, resultorientation and development of performance plans would also become significant. The fourth factor is delegation of public sector, competitiveness of market and delegation of authority to economic firms. Monopoly and single-polar economy may not meet the expectations of entrepreneur government. If a government intends to transform into an entrepreneur government, economic firms and delegation of authority must be prioritized. As a result, this factor would affect the realization of entrepreneur government. This factor along with the third factor lead to the fifth factor i.e. the government must provide a situation wherein private companies serve along with public sector companies. This would lead to competition in services and obviate economic recession in public organizations. When the benefits and revenues of a public sector organization become subject to threat, the organization must increase its performance and improve its services. Of course, the governments should not strengthen public organizations through application of massive administration budgets because this would manipulate the balance of competition in favor of public sector organizations. Exodus from cost-oriented thinking and alteration of budget policies towards performance-based budget is the sixth factor. In order to transform into an entrepreneur government, the administration must take off the disheveled garment of cost-orientation and take on major shifts in budget policies. The final factor in evaluation of public organizations is based on the outcome.

This would lead to more focus of public sector to the outcome i.e. performance. The amount of input in public sector is usually more than the output. Evaluation of output leads to output-orientation. An entrepreneur seeks lowering of expenses and frugality. According to schools of entrepreneurship, costly activities are not recommended while democracy is accompanied by expenses. Thus, collaboration and establishment of democratic government contradict entrepreneurship and its objectives. Tendency toward convergence exists in entire communities while on the other hand entrepreneurship transforms convergent society to a divergent one. An entrepreneur does not seek capacity building but looks for integration of capacities. Entrepreneur is not faithful to regulations and is a creative destroyer. Hence, response to people and beneficiaries is preferred to entrepreneurship. Development pattern based on entrepreneurship may resolve the weaknesses of public sector systems over time since it is a shift in essence which calls for sufficient time. If the objective of human resource development is to nurture the human capabilities and in case recession of resources leads to recession in development, human resources would undergo recession in public sector systems restrictive bureaucracy and policies. The slogan of development and revision of development plans during the first, second and third development plans contradicts human resources development since government has an integrated and restrictive system abundant with rules and regulations that do not yield human development. 


\section{Acknowledgement}

The authors would like to thank the anonymous referees for constructive comments on earlier version of this paper.

\section{References}

Ahmadpour, D. M., \& Moghimi, M. (2001). Role of entrepreneurial education on knowledge development of SMEs, Journal of Tadbir, 113.

Bygrave, W. D., \& Hofer, C. W. (1991). Theorizing about Entrepreneurship. Entrepreneurship theory and Practice, 16(2), 13-22.

Chou, S. W., \& Chang, Y. C. (2008). The implementation factors that influence the ERP (enterprise resource planning) benefits. Decision Support Systems,46(1), 149-157.

Drucker, P. (1970). Entrepreneurship in business enterprise. Journal of Business Policy, 1(1), 3-12.

Tzeng, G. H., \& Huang, J. J. (2011). Multiple attribute decision making: methods and applications. CRC Press.

Jahanshahloo, G. R., Lotfi, F. H., \& Izadikhah, M. (2006). Extension of the TOPSIS method for decision-making problems with fuzzy data. Applied Mathematics and Computation, 181(2), 15441551.

Denhardt, J. V., \& Denhardt, R. B. (2007). The new public service: Serving, not steering. ME Sharpe.

Kirzner, I. M. (1978). Competition and Entrepreneurship. University of Chicago press.

Lounsbury, M. (1998). Collective entrepreneurship: the mobilization of college and university recycling coordinators. Journal of Organizational Change Management, 11(1), 50-69.

Lynch, T.D., \& Cruise, P.L. (2006). Hand Book of Organization Theory and Management.The Philosophical Approach, $2^{\text {nd }}$ ed., Taylor \& Francis. New York.

Mierlo, J. G. A. (1996). Public entrepreneurship as innovative management strategy in the public sector: a public choice-approach (No. 020).

Osborne, D., Plastrik, P., \& Miller, C. M. (1998). Banishing Bureaucracy: the five strategies for reinventing government. Political Science Quarterly, 113(1), 168-169.

Osborne, D., \& Gaebler, T. (1992). Reinventing government: How the entrepreneurial spirit is transforming the public sector. 1992. HARV. BLACKLETTER J., 9, 163.

Prokopenko, J., \& Pavlin, I. (Eds.). (1991). Entrepreneurship development in public enterprises (Vol. 29). International Labour Organization.

Radfar, R., Pour Ebrahimi, A., \& Saeidikia, M. (2010). Entrepreneur government and role of information technology in its forming. Published articles, Islamic Azad University, Qazvin Branch.

Radfar, R., \& Saeidikia, M. (2010). Identification and studying the effective factors on information technology implementation for making an entrepreneur government. MA thesis, Islamic Azad University, Electronic Branch.

Sadler, R. J. (2000). Corporate entrepreneurship in the public sector: the dance of the chameleon. Australian Journal of Public Administration, 59(2), 25-43.

Say, J. B. (1836). A treatise on political economy: or the production, distribution, and consumption of wealth. Grigg \& Elliot.

Schumpeter, J. A. (1934). The theory of economic development: An inquiry into profits, capital, credit, interest, and the business cycle (Vol. 55). Transaction Publishers.

Wickham, P.A. (2000). 'Strategic Entrepreneurship' A decision making approach to new venture creation and management. Prentice Hall. 